\title{
Determinação da cobertura do solo por análise de imagens e redes neurais
}

\author{
Carlos A. A. Varella ${ }^{1}$, Francisco de A. de C. Pinto ${ }^{2}$, Daniel M. de Queiroz ${ }^{3} \&$ Darly G. de Sena Júnior ${ }^{4}$ \\ 1 Departamento de Engenharia da UFRRJ. Atualmente Doutorando em Engenharia Agrícola, bolsista PICDT/CAPES, \\ DEA/UFV. Viçosa, MG. CEP 36.571-000. Fone: (31) 3899-1881. E-mail: ds40146@correio.ufv.br (Foto) \\ 2 DEA/UFV. E-mail: facpinto@mail.ufv.br \\ 3 DEA/UFV. E-mail: queiroz@mail.ufv.br \\ 4 DEA/UFV. E-mail: darly@dea.ufv.br
}

Protocolo $027-5 / 3 / 2001$

\begin{abstract}
Resumo: Neste trabalho, desenvolveu-se um programa computacional para estimar a cobertura do solo, utilizando-se redes neurais (RN) treinadas por retropropagação do erro. Os dados para treinamento das $\mathrm{RN}$ foram obtidos de imagens digitais coloridas. Utilizou-se a razão entre a banda do verde e do vermelho para o pré-processamento das amostras de treinamento. Foram testadas cinco RN com arquiteturas 25- $n_{1}-n_{2}-2$. A arquitetura 25-20-10-2 foi a que apresentou melhor resultado sendo, portanto, utilizada no programa computacional classificador. O classificador apresentou índice de exatidão global de $82,10 \%$, cujo resultado mostra que redes neurais podem ser utilizadas em programas computacionais para separar feições onde a diferença entre a intensidade do brilho não permite a aplicação da técnica da limiarização.
\end{abstract}

Palavras-chave: visão artificial, plantio direto, processamento de imagem

\section{Soil cover determination using image analysis and neural networks}

\begin{abstract}
An image classification algorithm was developed to estimate the soil cover based on artificial neural networks (ANN) trained by back-propagation algorithm. The learning data sets were obtained from digital normalized images. Five ANN architectures of the type $25-n_{1}-n_{2}-2$ were tested. The architecture 25-20-10-2 presented the best result and therefore, it was used in the image classification program. The classification presented an overall accuracy of $82.10 \%$. This result shows that ANN may be applied for separating features when the pixel brightness does not provide enough information to apply the threshold technique
\end{abstract}

Key words: machine vision, no-tillage, image processing

\section{INTRODUÇÃO}

A cobertura do solo é um importante fator no processo da erosão, visto que reduz a energia de impacto das gotas de chuva sobre o solo, a velocidade e o volume de escoamento superficial e, conseqüentemente, o desprendimento das partículas e a capacidade de transporte de solo. A cobertura do solo é constituída pelo dossel da cultura e pelos resíduos vegetais, e sofre contínuas modificações a medida em que os resíduos se decompõem e a cultura se desenvolve. Desta forma, a quantificação desse parâmetro torna-se de grande importância para as pesquisas que estudam a infiltração e a erosão dos solos.

A cobertura do solo foi determinada por Sloneker \& Moldenhauer (1977) e Hartwig \& Laflen (1978) utilizando métodos de inspeção visual no campo. Laflen et al. (1981) descreveram e compararam esses métodos ("meterstick" e "line-transect") com o método do grid fotográfico, e concluíram que os três métodos estudados apresentaram resultados satisfatórios para a estimativa da cobertura do solo; contudo, deve-se observar que em cada um dos métodos mencionados, a determinação da cobertura do solo depende do julgamento do observador; além disso, esses métodos têm, como base, amostragens da cobertura do solo, as quais representam uma pequena porção da área estudada. Desta forma, um método menos subjetivo e que analise toda a superfície para determinar a cobertura do solo, seria mais vantajoso (Han \& Hayes, 1990b). Desde que os sistemas de imagens computadorizados se tornaram largamente utilizados e de baixo custo, diversos trabalhos têm sido realizados, com objetivo de se desenvolver uma técnica capaz de estimar a cobertura do solo por análise de imagens digitais. 
Meyer et al. (1988) testaram um sistema de visão artificial para estimar a cobertura do solo e concluíram que em solos úmidos a imagem apresentou melhor contraste que em solos secos. Utilizaram dois sistemas para aquisição das imagens, colorido e preto-e-branco, e compararam esses dois sistemas com o método do grid fotográfico. O sistema colorido utilizou apenas a banda do vermelho e o sistema preto-e-branco usou a banda do infravermelho próximo. Em ambos os sistemas utilizou-se da técnica da limiarização para segmentação da imagem mas nenhum algoritmo para reconhecimento de padrões; enfim, eles concluíram que o sistema colorido foi mais eficiente para determinar a cobertura do solo que o preto-e-branco.

Apesar do sistema colorido ter apresentado bons resultados no trabalho de Meyer et al. (1988) deve-se observar que esse sistema, com base na limiarização, assume que o solo e os resíduos vegetais apresentam intensidade de brilho distinta o suficiente para serem observados como padrões diferentes em uma imagem, o que se deve ao fato de que a limiarização classifica cada pixel individualmente, comparando a sua intensidade com um valor predefinido, o limiar. Quando a diferenciação da intensidade entre os objetos não ocorre, o método não se tem mostrado eficiente, visto que os resíduos se confundem com o solo, por apresentarem intensidades de brilho muito próximas e, desta forma, não podem ser separados apenas utilizando-se da técnica da limiarização.

Han \& Hayes (1990a) desenvolveram um procedimento para análise de imagem, com o objetivo de determinar a cobertura do solo (resíduos e/ou plantas) utilizando a informação textural da imagem. A imagem foi adquirida com câmara preto-e-branco e, posteriormente, digitalizada. Concluíram que o algoritmo foi capaz de quantificar a percentagem de solo coberto, com acurácia e sem influência do erro humano e, ainda, que a classificação de imagens utilizando a textura, pode separar resíduos/plantas do solo, mesmo quando estes apresentarem níveis de cinza com superposição aos níveis de cinza do solo descoberto. A análise textural, diferentemente da limiarização, que analisa cada pixel individualmente, avalia a correlação entre determinado pixel e os seus vizinhos. Desta forma, o valor da intensidade do pixel não é importante, mas, sim, a distribuição dessa intensidade, em uma região ao redor desse pixel.

Jayas et al. (2000) realizaram uma revisão bibliográfica sobre a aplicação de redes neurais (RN) como classificadores de imagens para aplicação na Engenharia Agrícola e concluíram que existe grande número de trabalhos publicados, utilizando diversas técnicas de processamento de imagens e de redes neurais para a classificação de imagens; porém a falta de integração entre essas duas técnicas ficou evidente. Finalmente, chegaram à conclusão que o conceito de processamento de imagens pode ser combinado com o de redes neurais classificadoras para serem usados em conjunto em sistemas de visão artificial para aplicações na agricultura. Lippmann (1987) revendo seis modelos de redes neurais, concluiu que esses modelos apresentaram grande potencial para reconhecimento de fala e imagem, visto que, nesses casos, são formuladas muitas hipóteses em paralelo e é exigido alto recurso computacional para o reconhecimento de padrões.

Vários estudos têm sido conduzidos com o objetivo de se comparar o desempenho e a classificação das redes neurais com os classificadores estatísticos (Huang \& Lippmann, 1987; Sethi, 1991; Luo et al., 1999). A maioria dos classificadores estatísticos tem como base a regra de decisão de Bayes, que assume uma distribuição normal da população. Apesar dessa regra de decisão ser muito simples, é difícil ser aplicada na prática porque a probabilidade posterior normalmente não é conhecida e, desta forma, deve ser estimada a partir das amostras (Jayas et al., 2000).

Na classificação com uso de redes neurais não é necessário fazer-se nenhuma consideração das funções de densidade de probabilidade; contudo, existe o problema de se definir a arquitetura da $\mathrm{RN}$, visto que não há nenhuma teoria para se determinar, a priori do treinamento, a arquitetura que melhor resultado apresentaria na classificação, mas se tem verificado que, com uma RN com apenas uma camada escondida, pode não ser capaz de aprender o suficiente para resolver determinado problema de classificação, enquanto redes com grande número de neurônios podem tender à memorização de padrões específicos no treinamento. A melhor forma de se estruturar uma rede é começar com poucas camadas escondidas e com poucos neurônios em cada camada. A complexidade da rede deve ser gradualmente aumentada, até que o resultado desejado seja obtido.

Redes neurais classificadoras têm sido utilizadas com sucesso para a solução de vários problemas ligados à atividade agrícola, como o florescimento e a maturação fisiológica da soja (Elizondo et al., 1994), a qualidade em grãos de milho (Liao et al., 1993), a detecção de rachaduras em ovos (Patel et al., 1995), a classificação de grãos para semente (Luo et al., 1999), a produtividade do milho (Liu \& Goering, 1999) e na direção de tratores agrícolas (Pinto et al., 1999).

Han \& Hayes (1990a) concluíram que a técnica textural de classificação de imagens elimina a necessidade de um sistema colorido de aquisição, porém a obtenção da informação textural de imagens digitais envolve uma quantidade considerável de recurso computacional, resultando em um longo tempo de processamento. Assim, as RN, utilizando-se como dados de entrada os valores numéricos de um pixel e seus vizinhos, apresentam-se como alternativa para a classificação de imagens onde os objetos não podem ser diferenciados utilizando-se da técnica da limiarização.

As RN podem requerer um tempo de processamento inferior aos métodos tradicionais de análise textural como, por exemplo, a matriz de co-ocorrência utilizada por Han \& Hayes (1990a). Acrescenta-se ainda que as $\mathrm{RN}$ podem ser implementadas em processamento paralelo aumentando substancialmente, a velocidade da classificação e possibilitando aplicações em tempo real.

Objetivou-se, com este trabalho, determinar a arquitetura de uma $\mathrm{RN}$ e desenvolver um programa computacional para se estimar a porcentagem de cobertura do solo, utilizando-se como dados de entrada na $\mathrm{RN}$ os valores numéricos pré-processados de imagens digitais coloridas.

\section{MATERIAL E MÉTODOS}

As imagens foram obtidas com uma câmara digital colorida Sony Mavica, modelo MVC-FD73. Cada imagem apresentava 
$480 \mathrm{H}$ x $640 \mathrm{~V}$ pixels, e foi adquirida de uma altura de aproximadamente 1,20 $\mathrm{m}$ do solo, apresentando um campo de visão no terreno de $0,93 \times 1,19 \mathrm{~m}$. Foram adquiridas 10 fotografias de uma área situada na localidade denominada Paraíso, no município de Viçosa, MG, mantida sob o sistema de plantio direto durante 3 anos consecutivos (1997-2000) com a cultura do milho (Zea mays L.) em rotação com a do feijão (Phaseolus vulgaris L.). O tipo de solo é um Terra Roxa Estruturada, apresentando $370 \mathrm{~g} \mathrm{~kg}^{-1}$ de argila, $490 \mathrm{~g} \mathrm{~kg}^{-1}$ de areia e $140 \mathrm{~g} \mathrm{~kg}^{-1}$ de silte, com textura argilo-arenosa. As imagens foram adquiridas após a colheita mecanizada do milho.

\section{Pré-processamento das amostras de treinamento}

Para treinamento das $\mathrm{RN}$ foram retirados, da imagem, blocos de $5 \times 5$ pixels que representassem cada objeto a ser classificado. Retiraram-se 20 amostras de treinamento da palha, 10 amostras da vegetação verde e 10 amostras do solo descoberto, de uma única imagem.

As amostras foram pré-processadas dividindo-se os valores numéricos da banda do verde pelos valores numéricos da banda do vermelho. Os valores numéricos das amostras pré-processadas foram obtidos por meio da Eq. 1 .

$$
\mathrm{VN}_{\mathrm{ijN}}=\frac{\mathrm{VN}_{\mathrm{ijG}}}{\mathrm{VN}_{\mathrm{ijR}}}
$$

\section{em que:}

$\mathrm{VN}_{\mathrm{ijN}}$ - valores numéricos das amostras pré-processadas

$\mathrm{VN}_{\mathrm{ijG}}$ - valores numéricos da banda do verde

$\mathrm{VN}_{\mathrm{ijR}}$ - valores numéricos da banda do vermelho

Adotou-se esse pré-processamento nas amostras devido o solo descoberto ter apresentado baixa reflectância na banda do verde e alta reflectância na banda do vermelho, vegetação verde com alta reflectância na banda do verde e baixa na banda do vermelho, enquanto a palha apresentou reflectâncias muito próximas nessas duas bandas; assim, após o pré-processamento as amostras de solo descoberto apresentaram valores entre 0,80 e 0,92, as de vegetação verde valores entre 1,07 e 2,91 e as amostras de palha valores entre 0,76 e 1,06.

Utilizando-se uma relação entre duas bandas, elimina-se a variação do valor numérico do pixel, devido à variação da iluminação natural durante o dia.

\section{Arquitetura e treinamento das RN}

Após pré-processadas, cada amostra foi convertida de uma matriz 5 x 5 pixels para um vetor coluna de 25 linhas, sendo que cada vetor constituiu uma das colunas da matriz, $25 \times 40$, para treinamento das RN. As colunas de 1 a 20 dessa matriz foram constituídas pelos vetores das amostras de palha, as de 21 a 30 pelos vetores das amostras de vegetação verde e as últimas 10 colunas pelos vetores das amostras de solo descoberto. Desta forma, as 30 primeiras colunas constituíram-se dos vetores de entrada para classificação da cobertura vegetal e as 10 últimas colunas pelos vetores, para classificação do solo descoberto.

Testaram-se cinco redes neurais de arquitetura $25-n_{1}-n_{2}-2$, isto é, 25 elementos no vetor da camada de entrada, $n_{1}$ e $n_{2}$ número de neurônios nas camadas escondidas e dois elementos no vetor da camada de saída e se utilizou a função de transferência tangente hiperbólica sigmóide, em ambas as camadas escondidas e a função de transferência logística sigmóide na camada de saída. Desta maneira, espera-se que, na saída da $\mathrm{RN}$, seja emitido um valor $(1,0)$ para um bloco que represente a cobertura do solo e um valor $(0,1)$ para blocos que representem o solo descoberto. As redes foram treinadas utilizando-se a técnica do gradiente descendente em lote pela retropropagação do erro. Nessa técnica, a rede utiliza uma procura para a solução ótima dos parâmetros livres na direção mais acentuada do gradiente negativo da curva do erro, sendo que os parâmetros livres são atualizados após o processamento de todos os dados de treinamento. O algoritmo da retropropagação do erro minimiza o erro quadrático médio (EQM) entre a resposta obtida de uma RN e a resposta desejada. A Tabela 1 apresenta a arquitetura das cinco RN testadas.

Tabela 1. Arquitetura das redes neurais testadas

\begin{tabular}{ccc} 
Rede Neural & $\mathrm{n}_{1}\left(1^{\mathrm{a}}\right.$ camada $)$ & $\mathrm{n}_{2}\left(2^{\mathrm{a}}\right.$ camada $)$ \\
\hline 1 & 5 & 3 \\
2 & 10 & 5 \\
3 & 20 & 10 \\
4 & 30 & 15 \\
5 & 40 & 20 \\
\hline
\end{tabular}

Cada uma das arquiteturas apresentadas na Tabela 1 foi treinada com 10.000 épocas e 10 inicializações aleatórias dos parâmetros livres e, para cada arquitetura testada, selecionouse a rede que apresentou o menor erro quadrático médio entre as 10 inicializações.

\section{Avaliação do treinamento das RN}

A escolha da RN para ser incorporada ao programa computacional de mensuração da cobertura do solo, foi escolhida com base no EQM apresentado no treinamento e na acurácia da classificação de uma amostra de validação; essas amostras foram obtidas de uma imagem diferente da usada para retirada das amostras de treinamento; essas amostras passaram pelo mesmo pré-processamento das amostras de treinamento.

\section{Determinação da cobertura do solo}

Desenvolveu-se um programa computacional para realizar a mensuração da cobertura do solo, nas imagens com a utilização da RN selecionada anteriormente. A imagem foi escaneada horizontalmente em blocos de 5 x 5 pixels; cada bloco, após o pré-processamento, era apresentado à RN, que o classificava como região com cobertura vegetal ou solo. No final, obteve-se uma imagem binária com valor 1 para os pixels classificados como solo descoberto e valor zero para os pixels classificados como cobertura do solo.

\section{Validação do programa computacional}

O programa foi validado pelo método da exatidão global da classificação proposto por Campbell (1987) que consiste no uso de uma matriz de erros ou tabela de contingência. Esse 
método, além de identificar o erro global da classificação para cada classe identifica, ainda, o erro entre classes. Essa matriz permite a identificação para cada classe dos erros de omissão e de comissão.

Selecionaram-se, inteiramente ao acaso, 100 pixels de referência em cada uma das 10 imagens originais, que foram comparados com a classificação do programa nas imagens binárias; a partir dessas informações, criou-se a matriz de erros

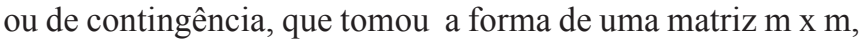
em que $\mathrm{m}$ é o número de classes analisadas. As linhas dessa matriz apresentavam a informação da imagem original e as colunas a informação sobre a imagem classificada. A partir dessa matriz, calcularam-se os erros de omissão, de comissão e o índice de exatidão global.

Os erros de omissão são definidos como os pixels omitidos de sua correta classe e assinalados em outra classe; os erros de comissão são os pixels erroneamente incluídos em uma classe, quando deveriam pertencer a outra classe; já o índice de exatidão global é o número de pixels corretamente classificado e dividido pelo número total de pixels de referência na imagem original, dado pela Eq. 2.

$$
\mathrm{EG}(\%)=\frac{\mathrm{Pc}}{\mathrm{Pt}} 100
$$

em que:

EG- índice de exatidão global

Pc - número de pixels corretamente classificado (diagonal da matriz de erros)

Pt - número total de pixels de referência na imagem original

\section{RESULTADOS E DISCUSSÃO}

\section{Arquitetura e treinamento das RN}

A Tabela 2 apresenta o menor erro quadrático médio obtido no treinamento para cada uma das arquiteturas testadas.

Tabela 2. Menor erro quadrático médio (EQM) obtido no treinamento das redes neurais $(\mathrm{RN})$

\begin{tabular}{ccc} 
RN & Arquitetura da RN & EQM \\
\hline 1 & $25-5-3-2$ & 0,074 \\
2 & $25-10-5-2$ & 0,085 \\
3 & $25-20-10-2$ & 0,070 \\
4 & $25-30-15-2$ & 0,070 \\
5 & $25-40-20-2$ & 0,069 \\
\hline
\end{tabular}

Observando-se a Tabela 2, verifica-se que a arquitetura (25-40-20-2) apresentou o menor erro no treinamento, porém esse valor foi aproximadamente igual aos valores obtidos para as arquiteturas (25-30-15-2) e (25-20-10-2) razão por que se procedeu à avaliação para essas três arquiteturas do erro quadrático médio e do número de testes corretos com as amostras de validação. A Tabela 3 apresenta os valores obtidos nessa avaliação.

Verifica-se, na Tabela 3, que a arquitetura (25-20-10-2) foi a que apresentou o menor erro quadrático médio e o maior número de testes corretos. Com base nesses resultados, selecionou-se esta arquitetura para fazer parte do programa computacional classificador.
Tabela 3. Erro quadrático médio (EQM) e número de testes corretos com as amostras de validação, das arquiteturas com menores EQM no treinamento

\begin{tabular}{cccc}
\hline RN & $\begin{array}{c}\text { Arquitetura } \\
\text { da RN }\end{array}$ & EQM & $\begin{array}{c}\text { No. de Testes } \\
\text { Corretos }\end{array}$ \\
\hline 3 & $25-20-10-2$ & 0,0891 & 36 \\
4 & $25-30-15-2$ & 0,1120 & 34 \\
5 & $25-40-20-2$ & 0,1000 & 34 \\
\hline
\end{tabular}

\section{Validação do classificador}

A Tabela 4 apresenta o índice de exatidão global, calculado por meio da Eq. 2, para cada uma das imagens classificadas pelo programa computacional.

Tabela 4. Índice de exatidão global (EG) para cada uma das imagens classificadas

\begin{tabular}{ccc}
\hline Número & Imagem & EG $(\%)$ \\
\hline 1 & mvc-56 & 92 \\
2 & mvc-57 & 90 \\
3 & mvc-58 & 71 \\
4 & mvc-59 & 90 \\
5 & mvc-60 & 82 \\
6 & mvc-61 & 86 \\
7 & mvc-62 & 81 \\
8 & mvc-63 & 76 \\
9 & mvc-64 & 73 \\
10 & mvc-65 & 80 \\
\hline
\end{tabular}

Verifica-se que a imagem mvc-56 foi a que apresentou o maior índice de exatidão global na classificação (92\%) mas é importante observar que as amostras de treinamento não foram retiradas dessa imagem e, sim, da imagem mvc-60, que apresentou índice de exatidão global de $82 \%$; assim, conclui-se que a RN (25-20-10-2) utilizada no classificador, além de apresentar um EG satisfatório apresentou, também, boa generalização, não apresentando indícios de memorização no treinamento.

A Figura 1 ilustra a imagem original mvc-056 e a imagem binária correspondente classificada pelo programa computacional.

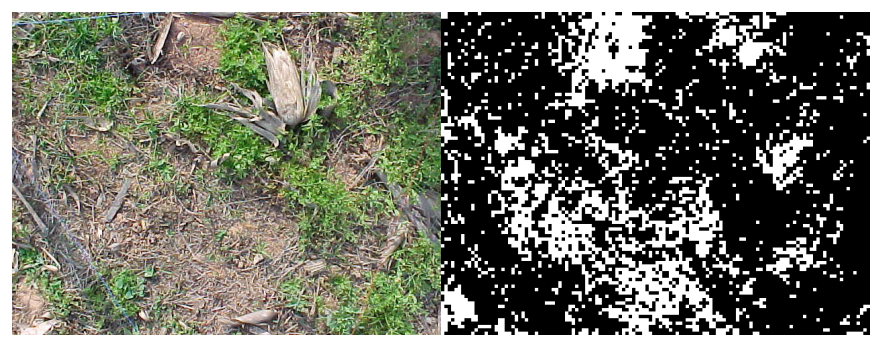

Figura 1. Imagem original mvc-056 e imagem binária correspondente classificada

A Tabela 5 apresenta a matriz de erros da classificação realizada pela rede neural (25-20-10-2) para as 10 imagens originais.

O índice de exatidão global médio obtido na classificação das 10 imagens foi de $82,10 \%$. Considerando-se que um valor em torno de $85 \%$ é usualmente adotado como aceitável para esse índice, conclui-se que o classificador apresentou resultado satisfatório na classificação das imagens analisadas, e que 
Tabela 5. Matriz de erros da classificação

\begin{tabular}{lcccc}
\hline Imagem de & \multicolumn{4}{c}{ Imagem Classificada } \\
\cline { 2 - 5 } Referência & Cobertura & Solo & Total & EO $^{*}(\%)$ \\
\hline Cobertura & 735 & 154 & 889 & 17,32 \\
Solo & 25 & 86 & 111 & 22,52 \\
Total & 760 & 240 & 1000 & \\
EC $^{*}(\%)$ & 22,52 & 17,32 & & \\
\hline * EO $(\%)$ - Erro de omissõ & EC $(\%)-$ Erro & &
\end{tabular}

redes neurais podem ser utilizadas para separar feições onde a diferença entre a intensidade do brilho não permite a aplicação da técnica da limiarização.

Verifica-se, na Tabela 5, que o erro de omissão (EO) médio para a cobertura do solo foi de $17,32 \%$, indicando que o classificador subestimou a cobertura do solo em aproximadamente $17 \%$, isto é, assinalou 154 pixels, que eram cobertura do solo na imagem original, como solo descoberto na imagem classificada. Observa-se, ainda, que o EO da cobertura é igual ao EC de solo e vice-versa, devido ao fato de que a classificação apresentou apenas duas classes. Verifica-se ainda, nessa tabela, que de um total de 1000 pixels analisados 889 foram de cobertura do solo, indicando predominância dessa classe nas imagens originais.

Apesar dos resultados obtidos neste trabalho terem sidos satisfatórios, recomenda-se que outros trabalhos, utilizando outros tipos de solos e outras coberturas vegetais, sejam realizados, possibilitando a identificação se uma RN de mesma arquitetura que a estudada, seria capaz de classificar, com a mesma exatidão global, a cobertura vegetal e o solo descoberto para outras condições.

A Tabela 6 apresenta a porcentagem de cobertura do solo determinada para cada uma das imagens analisadas pelo programa computacional desenvolvido.

Tabela 6. Porcentagem de cobertura do solo (CS) determinada pelo programa computacional para cada uma das imagens classificadas

\begin{tabular}{ccc}
\hline Número & Imagem & CS $(\%)$ \\
\hline 1 & mvc-56 & 74,24 \\
2 & mvc-57 & 85,37 \\
3 & mvc-58 & 37,58 \\
4 & mvc-59 & 80,75 \\
5 & mvc-60 & 80,05 \\
6 & mvc-61 & 85,16 \\
7 & mvc-62 & 41,01 \\
8 & mvc-63 & 65,23 \\
9 & mvc-64 & 48,17 \\
10 & mvc-65 & 65,34 \\
\hline Média & & 86,36 \\
\hline
\end{tabular}

\section{CONCLUSÕES}

1. A rede neural de arquitetura (25-20-10-2) foi a que apresentou o menor erro quadrático médio e maior acurácia nas amostras de validação.

2. O programa computacional apresentou índice de exatidão global médio na classificação de $82,10 \%$.

3. Classificadores com base em redes neurais podem ser utilizados para separar o solo descoberto da cobertura do solo, mesmo em situações em que a diferença entre a intensidade do brilho dessas feições não permite a aplicação da técnica da limiarização.

\section{LITERATURA CITADA}

Campbell, J.B. Introduction to remote sensing. New York: The Guilford Press, 1987, 551p.

Elizondo D.A.; McClendon R.W.; Hoogenboom G. Neural network models for predicting flowering and physiological maturity of soybean. Transactions of the ASAE, St. Joseph, v.37, n.3, p.981-988, 1994.

Han, Y.J.; Hayes, J.C. Soil cover determination by image analysis of textural information. Transactions of the ASAE, St. Joseph, v.33, n.2, p.681-686, 1990a.

Han, Y.J.; Hayes, J.C. Soil cover determination using color image analysis. Transactions of the ASAE, St. Joseph, v.33, n.4, p.1402-1408, 1990b.

Hartwig, R.O.; Laflen, J.M. A meterstick method for measuring crop residue cover. Journal of Soil and Water Conservation, Fairmont, W. Va, v.33, n.2, p.90-91, 1978.

Huang, W.Y.; Lippmann, R.P. Comparisons between neural net and conventional classifiers. IEEE International Conference on Neural Networks, 1, 1987, San Diego. Lexington, MA: MIT Lincoln Laboratory, 1987, v.4, p.485-493.

Jayas, D.S; Paliwal, J; Visen, N.S. Multi-layer neural networks for image analysis of agricultural products. Journal Agricultural Engineering Research, Silsoe, v.77, n.2, p.119-128, 2000.

Laflen, J.M.; Amamiya, M.; Hintz, E.A. Measuring crop residue cover. Journal of Soil and Water Conservation, Fairmont, W. Va, v.36, n.6, p.341-343, 1981.

Liao K.; Paulsen M.R.; Reid J.F.; Ni B.C.; Bonifacio-Maghirang E.P. Corn kernel breakage classification by machine vision using a neural network classifier. Transactions of the ASAE, St. Joseph, v.36, n.6, p.1949-1953, 1993.

Lippmann, R.P. An introduction to computing with neural nets. IEEE ASSP Magazine, v.4, n.2, p.4-22, 1987.

Liu, J. ; Goering, C.E. Neural network for setting target corn yields. St. Joseph, ASAE Paper No. 99-3040, 1999.

Luo X.Y.; Jayas D.S.; Symons S.J. Comparison of statistical and neural network methods for classification of cereal grains using machine vision. Transactions of the ASAE, St. Joseph, v.42, n.2, p.413-419, 1999 .

Meyer, G.E.; Stepanek, D.P.S.; Dickey, E.C. Electronic image analysis of crop residue cover on soil. Transactions of the ASAE, St. Joseph, v.31, n.3, p.968-973, 1988.

Patel, V.C.; McClendon, R.W.; Goodrum, J.W. Detection of cracks in eggs using computer vision and artificial neural networks. St. Joseph, ASAE Paper No. 95-3258, 1995.

Pinto, F.A.C.; Reid, J.F.; Zhang, Q.; Noguchi, N. Guidance parameter determination using artificial neural network classifier. St. Joseph, ASAE Paper No. 99-3004, 1999.

Sethi, I.K. Decision tree performance enhancement using artificial neural network implementation. Machine Intelligence and Pattern Recognition, Amsterdam, v.11, p.71-88, 1991.

Sloneker, L.L.; Moldenhauer, W.C. Measuring the amounts of crop residue remaining after tillage. Journal of Soil and Water Conservation, Fairmont, W. Va, v.32, n.5, p.231-236, 1977. 